\title{
International Collaboration for the Treatment of Recurrent Retroperitoneal Sarcoma: What Have We Learned from Trying to Fit a Square Peg in a Round Hole?
}

\author{
Barry W. Feig, MD \\ Department of Surgical Oncology, The University of Texas MD Anderson Cancer Center, Houston, TX
}

The diagnosis of "sarcoma" encompasses a large variety of different tumors (somewhere close to 75 different tumors), many of which have their own unique patterns of behavior based on their histologic tissue of origin. This is a principle that has been championed at the MD Anderson Cancer Center long before I ever arrived as a surgical oncology fellow. However, clinical researchers have been handicapped by the rarity of the disease process and by the inconsistencies in the treatment algorithms employed around the world. Although the study by Nessim et al. ${ }^{1}$ published in this edition of the Annals of Surgical Oncology does not provide any dramatic new data that might change the course of treatment and outcomes for patients with retroperitoneal sarcomas (RPS), it is an important study for a number of reasons. Most notably, within the framework under which this manuscript was developed may lie the answer (at least in part) for how the surgical sarcoma community can begin the process of making significant inroads into the improvement of the care and outcomes of patients with soft tissue sarcomas. The Transatlantic Australasian Retroperitoneal Sarcoma Working Group (TARPSWG) was established by a small group of sarcoma surgical oncologists with the goal of improving international collaboration in the treatment of patients with RPS. This was based on the premise that the most significant impediment to the advancement in the care and treatment of patients with sarcoma has been the

(C) Society of Surgical Oncology 2021

First Received: 9 December 2020

Accepted: 23 December 2020;

Published Online: 3 March 2021

B. W. Feig, MD

e-mail: bwfeig@mdanderson.org scarcity of quality data due to the rarity of the disease. Working collaboratively to standardize the treatment and care of these patients would allow for greater opportunities to accumulate data for quantitative analysis.

The aforementioned manuscript (Nessim et al. ${ }^{1}$ ) evaluates the surgical outcomes of patients who have undergone resection of recurrent RPS at 22 TARPSWG sarcoma centers in order to determine if it was safe to perform surgery in this patient population. Was this the most important question that needed to be addressed in the care of patients with recurrent RPS? Absolutely not. Will this paper change the surgical management of these patients? I see nothing in the manuscript to indicate that that would be the case. As an experienced, busy sarcoma surgeon, patients with recurrent RPS make up approximately twothirds of my surgical practice; however, excessive surgical morbidity is not something that I lie awake at night worrying about. It is important to document that, as a group, we are able to perform these surgeries safely, from a technical, surgical perspective. Most of the surgeons that perform these operations frequently believe they are more extensive and have always assumed them to be more difficult than surgery for a primary RPS. There is an undocumented assumption that the morbidity and mortality of the surgery for a recurrent RPS would be more significant than that seen after resection of a primary RPS. This is due to a variety of factors that are very difficult to quantify (such as extent of prior surgery, extent of adhesions, preoperative chemotherapy, preoperative radiotherapy, and nonanatomic dissection) but are generally felt to make any repeat abdominal surgery more difficult. Additionally, the thought process and decision making that goes into deciding whether to offer a patient with a recurrence a repeat abdominal surgery and if and how that operation will be beneficial to a patient are some of the most difficult 
decisions a surgical oncologist will have to make. In my mind, there is no question that the surgery for recurrent tumors is more extensive and creates more physiologic derangements for the patient than do similar surgeries for primary tumors.

In the current manuscript, that was found not to be the case. ${ }^{1}$ Not only was there no statistical difference in the morbidity or mortality when comparing the present data to the data previously published by the TARPSWG group after resection of a primary RPS, but as noted by the authors, the mortality was "quite lower" in the recurrent RPS group. Again, this flies in the face of surgical common sense. One possible explanation for this result is the inconsistency of the organizational model, as well as the methodology used to recruit participation of various sarcoma centers around the world. As previously mentioned, one of the initial goals of the TARPSWG group was to garner international collaboration to standardize the treatment algorithms for these patients. Unfortunately, it has been more difficult than expected to achieve this goal, most likely due to two reasons that long preceded the inception of TARPSWG, thus leaving this group with little control over either of these obstacles: the inability to standardize the classification of these tumors and the wide variability in consistency and experience of the sarcoma centers that submit clinical data for these studies. First, as previously mentioned, sarcoma represents a wide compendium of soft tissue tumors derived from different histologic origins. The behavior of many of these tumors can be extremely variable with each histologic subtype having its own unique, quirky behavior. This phenomenon has only recently been accepted by the international sarcoma community, but as mentioned previously, has been the cornerstone of the treatment of sarcomas at the MD Anderson Cancer Center since long before I began my career there in 1991. As a trainee and young faculty member, I was taught this academic/clinical principle by two of my mentors in sarcoma, Bob Benjamin (medical oncologist) and Raphael Pollock (surgical oncologist); this philosophy was based on the dedicated expert pathologic review that we were afforded to have at MD Anderson from Harry Evans. For example, if Dr. Evans reported a tumor as an MFH (malignant fibrous histiocytoma; currently termed a UPS, unclassified pleomorphic sarcoma), there was no reason to also add a label of "high grade," because all MFH's (unless they were myxoid) acted in an aggressive (high grade) manner (i.e., "a rose by any other name would smell as sweet"). ${ }^{2}$ In the early to mid-1990s, this philosophy was considered by the two or three other leading sarcoma centers in the United States not to be "state of the art" scientific evaluation of sarcoma patients. In fact, at the initial Connective Tissue Oncology Society meeting in Boston in 1995, I sat and listened in disbelief as an MD Anderson scientific presentation was publicly ridiculed as worthless, because histologic grade was not included as one of the variables in the outcome assessment. It took almost 10 years for other centers in the United States to recognize and validate this clinical approach and almost 20 years for the international community to agree that this is the appropriate strategy for classifying the behavior of this group of tumors. ${ }^{3,4}$ Unfortunately, this Hatfield versus McCoy's approach to clinical care resulted in fractioning within the international sarcoma community; as new specialized centers for the treatment of sarcomas increased at a relatively fast pace, these new centers had to decide as to which "team's" approach to classification they should follow (histology vs. grade driven), thus making it difficult to achieve an international consensus on the preferred treatment algorithms for RPS. Irrespective of that intellectual battle, once the international community agreed that sarcomas should be treated based on their histologic subtype, then the onus fell to the sarcoma pathologists to be immaculately consistent in assigning each tumor to a reproducible histologic diagnosis. Mostly, due to the rarity of sarcomas, but also in part due to this relatively recent universal adoption of the importance of histologic subtype in determining the behavior of each particular type of sarcoma, there are not as many pathologists around the world who are experienced enough in evaluating sarcomas to provide a reproducible pathologic diagnosis as is needed to be able to treat these tumors in a consistent manner. Without this pathologic consistency, it is difficult to be sure that we are comparing similar groups. This issue is addressed (perhaps inadvertently) in the current manuscript by limiting the number of pathologic diagnoses that were entered into the trial. However, even with this study limited to the three most commonly encountered sarcoma histologic subtypes, there can still be tumors that are difficult to classify, regardless of whether the reading pathologist is experienced or not. For example, it is not uncommon to see experienced pathologists disagree on the distinction between a retroperitoneal dedifferentiated liposarcoma with leiomyosarcomatous features and a primary retroperitoneal leiomyosarcoma. Another example of the pathologic complexity of these tumors is seen in the evolving classification of the retroperitoneal liposarcomas. In 1979, Harry Evans first described a less aggressive form of liposarcoma, which he termed "atypical lipoma." In a subsequent paper later that year, a more aggressive form of liposarcoma was identified, and it was classified as a dedifferentiated liposarcoma. ${ }^{6}$ In between these two extremes of clinical behavior, there have existed six to eight various combinations of other lipomatous subtypes. Even 40 years after the initial identification of the existence of multiple subtypes of lipomatous tumors that behave in extremely varied manners, the sarcoma community is still trying to 
refine this pathologic classification. The bias that this dilemma invariably creates, which can significantly interfere with the scientific evaluation of a data set, could be improved in real time if there was a central pathologic review by a small handful of pathologists who had the interest, knowledge, and experience to appropriately evaluate these tumors. In this manner, central pathologic review would at least provide consistency to the pathologic diagnosis within the realm of each specific trial.

The second potential source of bias (and therefore another potential obstacle to standardizing treatment algorithms for these tumors) is the variability of the participating institutions (both in number and experience) from study to study, particularly when precise, consistent pathology is an important feature of the experimental design. Because of this variability, we can never be certain that if results are compared across studies, that similar groups of patients are being evaluated. The results in any study can be significantly distorted (albeit unintentionally) by a potential imbalance in the number of sarcoma centers, as well as by the level of experience of each center that is submitting patient data to the study; there is no way to ensure that the same treatment algorithms, surgical approaches, and preoperative and postoperative care (just to name a few important clinical factors that are not tracked and controlled for when a center designates itself a sarcoma center of excellence) are applied consistently amongst what is a very diverse group. In the current manuscript, the dataset of eligible patients came from 22 different sarcoma centers. The authors in their discussion conclude that this procedure is "safe" as the morbidity and mortality are not statistically different from the groups' previously published evaluation of primary RPS. ${ }^{7}$ However, that prior study was based on patient data that was accrued from only eight institutions, and two of those institutions did not submit data for the current study. This enormous discrepancy in the institutions involved in these two studies most likely would make this direct comparison of morbidity and mortality invalid, because these two patient populations are potentially too dissimilar to be able to make a valid comparison. Studies involving more common disease processes may be able to overcome a discrepancy in direct comparisons, such as this. However, the rarity of sarcomas makes it more likely that this comparison could be negatively influenced due to the variability amongst the participating institutions.

Although the concept of international collaboration has innumerable potential benefits, there remains skepticism over the possibility of being able to come up with a consensus treatment plan for a disease process that is so disparate and has so many permutations, combinations, and rearrangements of clinical scenarios that it would be impossible to get all of the potential alternatives organized into a reasonably concise number of treatment algorithms. When the TARPSWG group first published their guidelines for the treatment of recurrent retroperitoneal sarcoma, I raised this concern in an invited editorial in the Annals of Surgical Oncology. ${ }^{8,9}$ Data presented in this manuscript support my previous hypothesis that it would be very difficult to create algorithms to cover a large enough percentage of all of the possible clinical scenarios to make that a useful strategy. ${ }^{1}$ In fact, $57.4 \%$ of the patients in the current study underwent an operation that did not fit into any category for assigning a resected organ score. I have serious patient safety concerns when greater than half the time the patient's care no longer fits into any available guidelines. If a patient is being treated at a "sarcoma center" that is on the lower end of experience, what happens to the patient when they no longer can be treated according to the guidelines, because there are no guidelines established for that patients' unique clinical scenario? According to the data presented by Nessim et al., ${ }^{1}$ that scenario happens greater than half of the time for patients with RPS. This means that there is significant potential for patients to "fall off" these preordained treatment pathways without safety net for them; there is no pathway available to get back on track. To be clear, the skepticism that is being expressed lies in the unlikely possibility of being able to "herd" this extremely varied group of tumors into a few useful, concise treatment algorithms. Historically, there is no question that international collaborative groups have been successful in other disease sites by asking difficult questions and using the power of their numbers to find appropriate answers. When any research group is performing data analyses, it is not only important that the correct question be asked but are asked in an appropriate scientific manner and that the data set is robust enough that there is a strong likelihood that the question will be answered. As mentioned previously, the primary question being addressed in this manuscript is reasonable, but it is not a "game-changing" question that if answered by the study would have a major impact on patient care or outcome. There are currently, and for the foreseeable future, many more important questions that the TARPSWG group is in an enviable position to address in patients with recurrent RPS that would more than likely have a direct, immediate impact on patient care.

Examples of important clinical questions for patients with recurrent RPS who desperately need an answer include: Should patients with multifocal disease ever be offered surgery? Who in this group of patients benefit from surgical resection? Who would benefit from getting chemotherapy before surgery? Who would benefit from getting radiotherapy before surgery? Who would benefit from chemotherapy and radiation therapy? How can we better stratify/characterize/identify which patients are 
going to benefit from resection of a recurrent RPS? These are the questions that keep me from sleeping at night and the same questions I had at the start of my career that I was certain would be readily answered long before my career was over. Unfortunately, that has not turned out to be the case. However, I truly do believe that it is possible that the answers to these questions will be found, quite possibly through international collaboration.

Recently, the Connective Tissue Oncology Society celebrated its 25th anniversary meeting. Dr. Shreyaskumar Patel, sarcoma medical oncologist from the MD Anderson Cancer Center, was giving the invited, introductory talk. He asked me for my input on what I thought were major advances in the surgical treatment of sarcoma during this time period. After thinking about an appropriate answer for several days, and even after talking with several colleagues, I realized that I was struggling to come up with a single significant event specific to the surgical treatment of sarcoma over the past 25 years. When we consider that President Richard M. Nixon declared a "war on cancer" in 1961, and we are only minimally closer to curing cancer now than we were at that time, it really should come as no surprise that when asked for my input, I was unable to come up with an answer that was satisfactory in my mind. Although this was a rather disappointing reality, I was able to achieve some solace in the knowledge that the possibility of this situation recurring in the next 25 years is rather slim. The interest in sarcoma is clearly growing, as seen by the exponential increase in the TARPSWG and CTOS memberships, with many young clinical investigators chomping at the bit to come up with better treatments and outcomes for sarcoma patients. With the proper guidance and availability of resources, I am confident that this group of young sarcoma surgical oncologists (Dr. Nessim,
Dr. Roland, Dr. Carmona, Dr. Fiore, Dr. Gyorki, Dr. van Houdt, et al.) are poised to soon become the next generational leadership of TARPSWG and will be able to answer many more of these "important" questions.

DISCLOSURE Dr Feig has nothing to disclose.

\section{REFERENCES}

1. Nessim C, Raut C, Calligaro D, et al. Postoperative morbidity after resection of retroperitoneal sarcoma: a report from the Transatlantic Australasian RPS Working Group (TARPSWG). Ann Surg Oncol. 2021. https://doi.org/10.1245/s10434-020-09445-y.

2. Shakespeare, William; Romeo and Juliette, Act 2, Scene 1.

3. Singer S, Atonescu A, Riedel E, et al. Histologic subtype and margin of resection predict pattern of recurrence and survival for retroperitoneal sarcomas. Ann Surg. 2003;238(3):358-70.

4. Gronchi A, Micelli R, Allard MA, et al. Personalizing the approach to retroperitoneal soft tissue sarcoma. Ann Surg Oncol. 2015;22(5):1447-54.

5. Evans HL, Soule EH, Winkelman RK. Atypical lipoma, atypical intramuscular lipoma, and well differentiated retroperitoneal liposarcoma. Cancer. 1979;43:574-84.

6. Evans HL. Liposarcoma: a study of 55 cases with a reassessment of its classification. Am J Surg Pathol. 1989;3(6):507-23.

7. MacNeill AJ, Gronchi A, Micelli R, et al. Postoperative morbidity after radical resection of primary retroperitoneal sarcoma; A report from the Transatlantic RPS Working Group. Ann Surg Oncol. 2017;24:688-9.

8. TARPSWG. Management of recurrent retroperitoneal sarcoma: a consensus approach from the Trans-Atlantic RPS working group. Ann Surg Oncol. 2016;23(11):3531-40.

9. Feig B, Benjamin R. Guidelines for the treatment of recurrent retroperitoneal sarcoma: are we trying to fit a round peg in a square hole. Ann Surg Oncol. 2016;23(11):4421-2.

Publisher's Note Springer Nature remains neutral with regard to jurisdictional claims in published maps and institutional affiliations. 